\title{
O SUJEITO/PESQUISADOR NA PESQUISA EM PSICOLOGIA CLÍNICA
}

\author{
The researcher in clinical psychology research
}

\author{
Juliane Callegaro Borsa ${ }^{a}$, Maria Lúcia Tiellet Nunes ${ }^{b}$ \\ a Psicóloga; Mestranda em Psicologia Clínica, PUCRS. Pesquisadora Bolsista do CNPq, pesquisadora do grupo de pesquisa \\ intitulado Formação, Avaliação e Atendimento em Psicoterapia Psicanalítica da Prof ${ }^{a}$. Maria Lúcia Tiellet Nunes, Porto Alegre, \\ RS - Brasil, e-mail: psicojuli@yahoo.com.br. \\ b Psicóloga; Doutora em Psicologia - Universidade Livre de Berlim. Professora titular da Faculdade de Psicologia, PUCRS. \\ Coordenadora do Programa de Pós-Graduação em Psicologia, FAPSI, PUCRS, Porto Alegre, RS - Brasil, e-mail: tiellet@pucrs.br.
}

\begin{abstract}
Resumo
O presente artigo é uma revisão teórica acerca dos aspectos pertinentes à pesquisa científica em Psicologia Clínica. Considerando a amplitude do tema, optou-se por apresentar um recorte, abordando, inicialmente, algumas discussões referentes aos diversos conceitos utilizados na definição de Psicologia Clínica. Em um segundo momento, serão focados alguns aspectos referentes à pesquisa em Psicologia Clínica, abordando as particularidades que a caracterizam, sobretudo quanto ao papel do sujeito/pesquisador neste processo. As considerações finais apontam para a relevância da pesquisa e do aprimoramento desta prática bem como a importância de conhecer as particularidades encontradas no âmbito da prática clínica, como é o caso da relação entre o pesquisador e o objeto. A Pesquisa em Psicologia e a Psicologia Clínica não podem ser entendidas como duas práticas distintas e não relacionadas.
\end{abstract}

Palavras-chave: Pesquisa em psicologia clínica; Psicologia clínica; Psicologia e ciência. 


\begin{abstract}
The present paper is a theoretical review about some aspects concerning scientific research in Clinical Psychology. Considering the breadth of the subject, it was decided to show just a cutting, initially approaching some discussions regarding the different concepts used in Clinical Psychology's definition. In a second moment, some aspects related to Clinical Psychology's research it will be focused, approaching the peculiarities that characterize it, mainly considering the role of the researcher in this process. Finally, the considerations point to the relevance of the research and the improvement of this practical as well as a bigger knowledge of the particularitities in the scope of the practical clinic, for example the relation between the researcher and the object. The Research in Psychology and Clinical Psychology cannot be understood as two practical distinct ones and not be related.
\end{abstract}

Keywords: Research in clinical psychology; Clinical psychology; Psychology and science.

\section{INTRODUÇÃO}

O nascimento oficial da Psicologia como ciência está marcado pela fundação do laboratório de Psicologia Experimental por Wilhelm Wundt, em Leipzig, Alemanha, em 1879. Desde então, este campo esteve atravessado por duas tradições epistemológicas e metodológicas: de um lado, a investigação naturalista do psiquismo (forma de trabalhar, comum no século XX, explorada pelos behavioristas e, hoje em dia, pelos cognitivistas e neurocientistas) que, quase sempre, foi desenvolvida nos institutos de pesquisa nas universidades; do outro lado, a investigação clínica do psiquismo que, ao contrário, originou-se, em sua grande parte, dos contextos práticos de atendimento psicológico que, somente com dificuldade, foi ingressando nos ambientes universitários (Figueiredo, 1995). Em decorrência disso, até os dias de hoje, a investigação no âmbito da Psicologia Clínica é questionada quanto a sua legitimidade. A investigação na clínica psicológica remete-se ao campo da Psicologia Clínica eànecessidade de compreender sua origem e definição.

O termo Psicologia Clínica foi cunhado pelo psicólogo americano Lightner Witmer, o qual, após trabalhar com Cattell e Wundt e receber seu título de doutor na Alemanha, em 1892, regressou para a Universidade da Pensilvânia, onde passou a trabalhar com Psicologia Experimental. Seu interesse esteve voltado para a aplicação prática de achados de experimentos realizados nos laboratórios. Witmer estabeleceu a primeira Clínica de Psicologia, marcando o nascimento desta, na Pensilvânia, em 1896. Nesse mesmo ano, Witmer utilizou, pela primeira vez, o termo Psicologia Clínica e ministrou o primeiro curso de Psicologia Clínica. Na prática clínica, foi possível, ao mesmo tempo, atender ao público e formar estudantes para a prática clínica. Witmer defendia a Psicologia Clínica como um campo de estudo e de intervenção cujo objetivo seria o de examinar as aptidões dos sujeitos e suas deficiências com vistas à generalização destes achados. Também fundou a revista The Psychological Clinic, em 1907. Nessa publicação, havia uma descrição sobre a prática da Psicologia Clínica como sendo o exame e o tratamento de características individuais - não necessariamente anormais, associadas ao fenômeno do desenvolvimento (Nietzel, Bernstein \& Milich, 1998; Cullari, 2001, Sundbergs, Winebarger \& Taplin, 2002). Nasce, assim, o primeiro conceito de Psicologia Clínica.

De seu momento histórico inicial, com Witmer, até os tempos atuais, definições do campo de estudo e atuação da Psicologia Clínica apontam para alguns elementos recorrentes. Para iniciar a apresentação de algumas definições de Psicologia Clínica, serão utilizados, num primeiro momento, os conceitos e definições extraídos de dicionários de Psicologia e áreas afins.

Stedman (1911/1996) define a

Psicologia Clínica como um ramo da Psicologia especializado tanto na descoberta de novos conhecimentos como na aplicação da arte e da Ciência da Psicologia em indivíduos com distúrbios emocionais ou comportamentais.

Campbell (1970/1986) conceitua a Psicologia Clínica como um conjunto de princípios psicológicos específicos que lidam com os 
distúrbios e problemas mentais, emocionais, comportamentais e de desenvolvimento; o autor também traz a pesquisa como uma atividade relacionada à prática clínica.

Para Cabral \& Nick (1974/1997), a Psicologia Clínica é um ramo da Psicologia que tem por finalidade básica o desenvolvimento e a aplicação das técnicas de diagnóstico e psicoterapêuticas para a identificação e tratamento de distúrbios do comportamento.

Conforme Doron \& Parot (1991/1998), a Psicologia Clínica é o estudo aprofundado de casos individuais. De acordo com os autores, o termo "Clínica" originou-se da palavra grega kliné (leito), o que denota a atividade clínica como a relação do médico que, à cabeceira do paciente, examina as manifestações da doença para fazer um diagnóstico, um prognóstico e prescrever um tratamento.

Segundo Roudinesco \& Plon (1997/ 1998), entende-se por Psicologia Clínica a prática terapêutica fundamentada na entrevista direta e no exame de casos a partir da observação das condutas individuais.

Stratton \& Hayer (1994/2002) a definem como o ramo da Psicologia aplicada que está interessada no emprego dos insights e métodos obtidos da Psicologia teórica e da experiência clínica a fim de dar assistência às pessoas com problemas na vida ou com dificuldades psicológicas. Os autores também apontam que a Psicologia Clínica faz uso de técnicas específicas de avaliação e tratamento.

Além dos dicionários de Psicologia e áreas afins, outros textos sobre o tema se inserem nesta tentativa de conceituar a Psicologia Clínica. Cullari (2001) aponta que o objetivo da Psicologia Clínica é compreender o indivíduo e a conduta humana na sua totalidade.

Sundbergs et al. (1962/2002) trazem a Psicologia Clínica como o estudo dos problemas humanos por meio de conhecimentos e habilidades específicas, os quais almejam auxiliar os indivíduos na busca por uma melhor qualidade de vida.

Nietzel, Bernstein \& Milich (1987/1998) trazem a definição desta prática como o estudo e a avaliação do indivíduo em particular e de suas características específicas, sobretudo daqueles que estão em sofrimento psíquico ou com problemas de ajustamento. Os autores mencionam, também, como prática da Psicologia Clínica, a condução de pesquisas sobre o comportamento e processos mentais do indivíduo.

Por fim, segundo a American Psychological Association (APA), a Psicologia Clínica é o campo da Psicologia que integra ciência, teoria e prática para entender, predizer e aliviar o desequilíbrio, a invalidez e o incômodo; promove a adaptação humana, o ajuste e o desenvolvimento pessoal. De acordo com a APA, a Psicologia Clínica está focada nos aspectos intelectuais, emocionais, biológicos, psicológicos, sociais e do comportamento humano, que funcionam de diferentes formas nas diversas culturas e em todos os níveis socioeconômicos (Cullari, 2001; Sundbergs et al., 2002).

De acordo com as definições trazidas dos dicionários e demais textos, foi possível encontrar as seguintes informações: o conceito de Psicologia Clínica está, em síntese, relacionado ao ato de diagnosticar, examinar, avaliar e tratar o indivíduo, mediante técnicas e princípios específicos e característicos da Psicologia Clínica. Conhecer e compreender esses métodos e essas técnicas é extremamente importante, porém este tema não será apresentado neste artigo, por se tratar de um assunto relevante e amplo em demasia para ser significativamente discutido nestas breves linhas.

Outro aspecto importante é que parte das definições trouxe, em algum momento, a Psicologia Clínica como uma parte da Psicologia que visa aos casos individuais representativos de alguma anormalidade ou sofrimento psíquico, ou seja, daqueles indivíduos com problemas e distúrbios mentais, emocionais ou de comportamento. Embora a maioria dos conceitos de Psicologia Clínica aborde esta prática como intervenção, avaliação ou tratamento de indivíduos com sofrimento psíquico, vale lembrar que muitas abordagens teóricas utilizadas na prática clínica não estão centradas, apenas, na doença ou patologia; enfatizam, também, a idéia de prevenção de ênfase aos aspectos saudáveis do indivíduo.

Nesse contexto, ainda, foi possível observar que os diversos conceitos trazidos na literatura não mencionam uma informação relevante no âmbito da Psicologia Clínica e que diga respeito às diversas abordagens e referenciais teóricos que podem ser utilizados. Embora seja mais adequado falar "Das Psicologias Clínicas", optamos, neste artigo, por tentar discutir a Psicologia Clínica como um conceito único, 
estabelecido a partir dos critérios de tratamento, avaliação e, ainda, da prevenção, conforme proposto pelos autores acima mencionados.

Do mesmo modo, foi possível observar, até aqui, que poucos autores trazem a pesquisa como uma prática inerente à Psicologia Clínica, o que denota um interesse limitado da pesquisa neste âmbito. Embora tenha recebido pouca menção, a pesquisa, além de avaliação e tratamento, é uma atividade importante que, por sua vez, cumpre o papel de subsidiar e aprimorar a prática clínica (Nietzel et al., 1998).

\section{DESENVOLVIMENTO}

De acordo com as Atribuições Profissionais do Psicólogo no Brasil, redigidas pelo Conselho Federal de Psicologia e enviadas ao Ministério do Trabalho em 1992, são trazidos os seguintes aspectos: o campo de atuação em Psicologia Clínica ocorre em diversos âmbitos, utilizando o enfoque preventivo ou curativo, individualmente ou em equipe, por meio das várias abordagens teóricas; o psicólogo clínico realiza a pesquisa, o diagnóstico, o acompanhamento psicológico e a intervenção psicoterápica; a prática da pesquisa é trazida em vários momentos como uma atividade relevante da prática em Psicologia Clínica, seja visando à ampliação e à construção do conhecimento teórico aplicado, seja na elaboração de programas aplicados de pesquisa.

Apesar de a pesquisa em Psicologia Clínica ser considerada uma prática relevante, observa-se que poucos profissionais se dedicam a ela. Em pesquisa apresentada no Relatório Final do Conselho Federal de Psicologia, realizada pelo Instituto de Pesquisa de Opinião e Mercado para o CFP, em 2001, constatou-se que, dos 1200 sujeitos entrevistados, $54,9 \%$ dos psicólogos brasileiros são psicólogos clínicos. No entanto, do número total de participantes, apenas $0,6 \%$ exerce atividades de pesquisa. Esses dados foram corroborados na Pesquisa de Opinião com Psicólogos Inscritos no Conselho Federal de Psicologia, realizada em 2004, pelo IBOPE, para o próprio CFP, com 2000 participantes. Nessa pesquisa, constatou-se que $55 \%$ dos entrevistados desenvolvem atividades em Psicologia Clínica.
Dentre o número total de participantes, porém, apenas 5\% exercem atividades de pesquisa em Psicologia. Embora a pesquisa ainda seja pouco praticada entre os psicólogos clínicos, observouse que houve aumento desta prática, considerando a primeira pesquisa em 2001 e a última, em 2004.

A partir dos dados apresentados nessas pesquisas, é possível observar um número significativo de profissionais de Psicologia atuantes na Clínica. Por outro lado, constata-se um percentual muito pequeno de profissionais engajados em atividades de pesquisa. Considerando que $46 \%$ dos psicólogos concluíram seus cursos a partir de 1996, encontrou-se um percentual muito pequeno quanto à busca por cursos de mestrado e doutorado: apenas 7\% ingressam nos programas de Mestrado e 2\% nos programas de Doutorado. Analisando essas informações, levanta-se a questão: De que maneira a prática clínica alimenta a pesquisa, e de que forma a pesquisa contribui na prática em Psicologia Clínica?

A Psicologia Clínica consiste em um modo de atuação na Psicologia que traz inúmeras discussões e questionamentos acerca da sua eficácia e eficiência, seu campo de atuação e suas práticas (Feijoo, 2004). A pesquisa científica em Psicologia Clínica direciona-se ao campo do procedimento clínico com seus objetivos, seus métodos e seu apoio nas práticas e no campo da pesquisa definida como processo válido de produção de conhecimento. Neste contexto, é importante indagar sobre o lugar que ocupa a pesquisa clínica entre a prática clínica de intervenção e a pesquisa científica em Psicologia (Allones, 2004).

A idéia de que a pesquisa em Psicologia Clínica pode ser útil para a prática clínica não é recente. De acordo com Elliot \& Morrow-Bradley (1994), pesquisa e prática existem em uma espécie de diálogo uma com a outra. Esse diálogo pode ser tenso e improdutivo, com cada lado desvalorizando ou tentando dominar o outro. Contudo, esse diálogo pode proporcionar uma contribuição mútua entre as duas práticas. Por fim, segundo os autores, o maior problema não é exatamente a lacuna entre a Clínica e a Pesquisa, mas o fracasso na realização de um diálogo mutuamente desafiador e facilitador.

No contexto da teorização psicanalítica, a clínica e a pesquisa se articulam de forma 
conflituosa, pois o conhecimento de seu objeto, o aparelho psíquico ${ }^{1}$, está sujeito a toda espécie de desequilíbrios, vicissitudes e suspeitas. A pesquisa em Psicologia Clínica não é uma mera aplicação de conhecimentos. Ela se constrói numa dialética permanente entre teoria, método e prática. Seu ponto de partida é o posicionamento dialógico do pesquisador em relação ao sujeito, à valorização da subjetivação emergente e às condições concretas de onde e como este saber e relação ocorrem (Vieira Filho \& Teixeira, 2003).

Inúmeras discussões ocorrem em relação à pesquisa em Psicologia Clínica, considerando um aspecto bastante significativo que trata das características do pesquisador que, neste contexto, acabam sendo as mesmas de seu objeto de pesquisa, ou seja, possuem o mesmo status. Para melhor compreender esta dinâmica, definirse-á, a seguir, o sujeito/objeto como aquele que se situa no lugar de "pesquisado" e sujeito/ pesquisador aquele que se coloca no papel de investigador clínico.

Pensando sobre o sujeito/pesquisador, pode-se dizer que a Psicologia sofre influências internas e externas que interferem na visão do sujeito/pesquisador e na construção da pesquisa. Isso porque, de forma geral, a experiência deste pesquisador está envolvida na investigação, o que implica importantes particularidades no controle das variáveis, tornando a pesquisa científica complexa. Na Psicologia Clínica, esta complexidade fica ainda mais evidente, pois implica um processo personalizado e dinâmico de investigação (Batista Pinto, 2004).

Pensando sobre o sujeito/objeto da pesquisa em Psicologia Clínica, é possível pensar que este é a substância a ser estudada e, como tal, constitui-se a partir de propriedades e extensão passíveis de mensuração. Este sujeito consiste num objeto simplesmente presente em um espaço determinado. Trata-se de um objeto encerrado em si mesmo, não apreensível pela visão ou pelo tato (Feijoo, 2004). Na Psicologia, tudo o que ocorre com o sujeito é estudado pelos próprios recursos de um sujeito, diferindo, portanto, dos estudos epistemológicos das ciências formais (Campos, 1973). Dessa forma, entende-se a Psicologia Clínica como uma ciência cujo objeto é o sujeito e as próprias coisas do sujeito, sendo, também, o pesquisador um sujeito que traz algo que lhe é inerente.

A partir desse entendimento, destaca-se o estudo da implicação do sujeito/pesquisador como um dos momentos significativos da pesquisa em Psicologia Clínica. Em vez de considerá-lo como independente da construção de seu sujeito/ objeto de estudo, entende-se que se deve incluílo como parte integrante do processo. Construir um conhecimento defensivo em relação às emoções, às opiniões diferenciadas dos padrões comuns, aos valores pessoais e de grupo significa, antes de tudo, desimplicar o sujeito/pesquisador de seu saber/fazer (Vieira Filho \& Teixeira, 2003).

A epistemologia específica desse tipo de investigação interfere no objeto a ser investigado e, por isso, não há neutralidade possível. A pesquisa está sempre associada à realidade e à subjetividade do pesquisador. Portanto, há influências diretas e indiretas, conscientes e inconscientes do pesquisador na própria ciência que produz, tendo em vista que a sua produção sofre interferências de sua história e de seu funcionamento psicológico. A partir desse ponto de vista, pode-se dizer que o sujeito/objeto estudado é co-produzido pela subjetividade do sujeito/pesquisador. Essa afirmativa contraria os pressupostos da ciência positivista, que se baseia na eliminação da subjetividade na elaboração do conhecimento científico, recorrendo, assim, às noções de "objetividade" e "neutralidade". A subjetividade do sujeito/pesquisador influencia a compreensão que ele tem do sujeito/objeto. Por essa razão, o sujeito/pesquisador vivencia a investigação com seus valores, emoções, sentimentos e desejos que lhe são suscitados no contato com a realidade estudada e no interior de uma rede de relações e instituições sem neutralidade (Vieira Filho \& Teixeira, 2003).

Mesmo considerando a idéia de um objeto subjetivo, o conhecimento gerado pela pesquisa em Psicologia Clínica não se caracteriza como ilegítimo. A investigação científica em Psicologia Clínica pode proporcionar um amadurecimento e um desenvolvimento das técnicas e dos procedimentos empregados nos tratamentos psicológicos, visando à saúde mental dos indivíduos. Quanto mais a área clínica se

\footnotetext{
Traz-se como exemplo o aparelho psíquico, objeto de investigação da pesquisa em Psicologia Clínica cujo referencial teórico é a Psicanálise e as teorias contemporâneas advindas dela.
} 
desenvolve, e isso só é possível com o desenvolvimento de pesquisas, mais possibilidades de intervenção são descobertas. Sendo assim, a prática clínica pode e deve subsidiar a pesquisa que, por sua vez, subsidia a prática, estabelecendo-se um estreito laço entre esses dois aspectos, que se mostram indissolúveis. Portanto, existe uma relação circular, em que a construção do conhecimento clínico parte de considerações teóricas e aplicadas para retornar a elas, ou seja, a pesquisa deve partir e retornar às questões clinicamente relevantes (Tavares, 1995; Castro, 1999). Na pesquisa em Psicologia Clínica, a produção do conhecimento se constitui como o objetivo principal do sujeito/pesquisador. Tratase de tomar a prática por objeto, a fim de transformá-la e aprimorá-la em um tempo ulterior, graças aos resultados da pesquisa (Allones, 2004).

A partir desses apontamentos, pode-se pensar na pesquisa não mais como uma prática que, necessariamente, tenha que seguir o rigor estabelecido pelo positivismo, por meio de uma neutralidade utópica quanto ao papel do pesquisador. Conforme Ewald, Mancebo, Uziel \& Prestrelo (2004), fazer ciência não é simplesmente seguir um caminho previamente estabelecido, mas sim refletir sobre o mundo a partir de parâmetros inesperados, este modo normal (grifo no original) de fazer ciência e, ainda assim, estar produzindo narrativas e conhecimentos extremamente relevantes para a compreensão das coisas do mundo vivido.

\section{CONSIDERAÇÕES FINAIS}

Após essa breve reflexão, a partir da contribuição de alguns autores sobre a pesquisa em Psicologia Clínica, compreende-se a relevância $\mathrm{da}$ pesquisa a fim de proporcionar um aprimoramento desta prática e um maior conhecimento dos fenômenos e descobertas que só são encontrados no âmbito da prática clínica. Algumas especificidades são relevantes no contexto da Psicologia Clínica e implicarão na pesquisa, como é o caso da relação sujeito/ pesquisador e sujeito/objeto.

Muito embora se saiba que a neutralidade tão desejada pela ciência positivista do século passado deixa de existir no momento em que o pesquisador coloca os pés no campo a ser pesquisado, ainda há quem pense que a intervenção do pesquisador junto aos sujeitos pesquisados faz com que a pesquisa perca seu valor de verdade (Andrada, 2003).

Para Vieira Filho e Teixeira (2003), porém, a implicação do sujeito/pesquisador coloca-se, então, como um momento significativo na pesquisa em Psicologia Clínica. Em vez de considerá-lo como independente da construção do seu sujeito/objeto de estudo, entende-se que se deve incluí-lo como parte integrante do processo cognoscitivo. Para os autores, construir um conhecimento defensivo em relação às emoções, às opiniões diferenciadas dos padrões comuns, aos valores pessoais e de grupo significa, antes de tudo, desimplicar (grifo do autor) o clínico de seu saber/fazer.

A Pesquisa em Psicologia Clínica configura-se como um espaço repleto de particularidades quanto aos procedimentos técnicos e metodológicos. Embora essa conjuntura faça da pesquisa em Psicologia Clínica um tema polêmico e aparentemente inesgotável, é possível compreender que a Pesquisa em Psicologia e a Psicologia Clínica não podem ser entendidas como duas práticas distintas e não relacionadas.

Conforme os apontamentos de Elliot \& Morrow-Bradley (1994), ambas as práticas Clínica e Pesquisa - podem favorecer uma a outra. Os pesquisadores serão melhores na medida em que fizerem uso de suas habilidades clínicas; os clínicos podem ser melhores na sua prática na medida em que tiverem um maior envolvimento com a Pesquisa. Assim, Psicologia Clínica e Pesquisa em Psicologia podem se ajudar mutuamente para proporcionar medidas práticas mais eficazes que ajudem o paciente na sua reabilitação. Para isso, torna-se necessário somar as qualidades de ambos e mitigar as deficiências um do outro, mesclando conhecimento para, assim, criar um produto final que tenha utilidade e significado à prática em Psicologia Clínica.

A pesquisa em Psicologia Clínica deve proporcionar o desenvolvimento de metodologias que assumam ser situações de construção de sentido e estejam voltadas para favorecer a melhoria da condição humana, mesmo que tais trabalhos contenham riscos de imprecisões (Ferreira, 2002). Cada vez se torna mais relevante se pensar na interface da Pesquisa e da Psicologia Clínica, como 
objeto de estudo em si, de forma a proporcionar respostas às questões oriundas do fazer clínico. Como bem pontuou Guirado (1997), discutir este tema interessa, diretamente, a quem vive o desafio de ocupar esses dois diferentes lugares no exercício da Psicologia: a pesquisa e a Psicologia Clínica.

\section{REFERÊNCIAS}

Allones, C. R. (2004). Os procedimentos clínicos nas ciências humanas: Documentos, métodos, problemas. São Paulo: Casa do Psicólogo.

Andrada, E. G. C. (2003). Intervir ou não Intervir, Eis a Questão! Refletindo Sobre a Intervenção do Pesquisador no Campo Pesquisado. Psicologia Ciência e Profissão, 23(2), 42-47.

Batista Pinto, E. (2004). A pesquisa qualitativa em Psicologia Clínica. Psicologia USP, 15(1-2), 71-80.

Cabral, A. \& Nick, E. (1997). Dicionário técnico de psicologia. São Paulo: Cultrix.

Campbell, R. (1986). Dicionário de psiquiatria. São Paulo: Martins Fontes.

Campos, D. M. S. (1973). Introdução à pesquisa em psicologia. Petrópolis: Vozes.

Castro, P. F. (1999). Reflexões em psicologia e ciência: uma análise da pesquisa aplicada à psicologia clínica. Psicologia: Teoria e Prática, 1(1), 3-13.

Cularri, S. (2001). Fundamentos de psicologia clínica. México: Pearson Education.

Doron, R. \& Parot, F. (1998). Dicionário de psicologia. São Paulo: Ática.

Elliot, R. \& Morrow-Bradley, C. (1994). Developing a Working Marriage Between Psychotherapists and Psychoterapy Researchers: Identifying Shared Purposes. In P.F., Talley, H. H., Strupp, \& S. Butler. Psychotherapy research and practice Bridging the Gap. New York: BasicBooks.

Ewald, A.; Mancebo, D; Uziel AP; Prestrelo, E. (2004). A Questão da Cientificidade: Novos Paradigmas. Estudos e Pesquisas em Psicologia, 4(2).
Feijoo, A. M. L. C. (2004). A psicologia Clínica: técnica e techne. Psicologia em Estudo, 9(1), 87-93.

Ferreira, R. F., Calvoso, G. G., \& Gonzales, C. B. L. (2002). Caminhos da Pesquisa e a Contemporaneidade. Psicologia: Reflexão e Crítica, 15(2), 243-250.

Figueiredo, L. C. (1995). Investigação em Psicologia Clínica. In Conselho Federal de Psicologia. Psicologia no Brasil: Direções epistemológicas. Brasília: Conselho Federal de Psicologia.

Guirado, M. (1997). Vértices da Pesquisa em Psicologia Clínica. Psicologia USP, 8(1), 143-155.

Nietzel, M., Bernstein, D. \& Milich, R. (1998). Introduction to Clinical Psychology. ( $5^{a}$ ed). New Jersey: Upper Saddle River.

Pesquisa de Opinião com Psicólogos Inscritos no Conselho Federal de Psicologia. (2001). In Conselho Federal de Psicologia: Recuperado em 23 de Jul. de 2007, da Pol (Psicologia Online): http://www.pol.org.br/ publicacoes/pdf/Pesquisa_IBOPE.pdf

Relatório Final do Conselho Federal de Psicologia. (2000) In Conselho Federal de Psicologia. Recuperado em 20 de Jun. de 2007, da da Pol (Psicologia Online): http:// www.pol.org.br/publicacoes / pdf/ Pesquisa_WHO.pdf

Roudinesco, L., \& Plon, M. (1998). Dicionário de psicanálise. Rio de Janeiro: Zahar.

Stedman Dicionário Médico. (1996). (S. A. Teixeira, Trad.). (25 $5^{a}$ ed). Rio de Janeiro: Guanabara-Koogan.

Stratton, P., \& Hayer, N. (2002). Dicionário de Psicologia. São Paulo: Pioneira.

Sundberg, N., Winebarger, A., \& Taplin, J. (2002). Clinical psychology: Evolving theory, practice and research. ( $4^{\mathrm{a}}$ ed). New Jersey: Upper Saddle River.

Tavares, M. (1995). O papel do clínicopesquisador para o desenvolvimento da psicologia clínica. Psicologia: Reflexão e Crítica, 8(2), 169-180. 
Vieira Filho, N. G., Teixeira, V. M. S. (2003). Observação clínica: estudo da implicação psicoafetiva. Psicologia em Estudo, 8(1), 23-29.

Recebido: 09/10/2007

Received: 10/09/2007

Aprovado: 16/11/2007

Approved: 11/16/2007 